\title{
Beyond Cassini
}

The triumph of the spacecraft's arrival in orbit around Saturn last week heralds four years of outstanding research. But the longer-term ambitions of NASA and planetary researchers signal trouble ahead.

$\mathrm{F}$ ew fields of science require more patience than planetary exploration. The scientists who built instruments for the Cassini-Huygens spacecraft now orbiting Saturn began planning for the mission in the 1980s. They worked feverishly to meet a 1997 launch date, then waited seven long years for the first results.

Early signs suggest that the pictures and data now streaming back from Saturn will be worth the wait. Cassini's powerful instruments have already shown new detail in the planet's ring system and revealed the icy satellite Phoebe to be a remnant from the first days of the Solar System. The large moon Titan is proving more mysterious than ever, strangely devoid of the large oceans that many suspected would lie underneath its clouds. For planetary scientists, it looks to be an exciting and surprising four years.

Several of the Cassini investigators are veterans of pioneering missions in the 1980s and 1990s, and can rightly be called the first generation of planetary explorers. What's the outlook for the next generation? Mostly sunny, but with clouds gathering on the horizon.

In recent years, NASA has created a balanced, healthy planetaryexploration programme, at least on paper. Ever more sophisticated Mars probes launch every two years and are producing results. The Discovery programme - modest-sized missions proposed by the research community - has been a scientific and budgetary success. The next spacecraft in the series, MESSENGER, will lift off this month to study the Solar System's innermost planet, Mercury.

As for the outer Solar System, New Horizons will launch in 2006 to the last unexplored planet, Pluto. And NASA is in the early stages of planning its Jupiter Icy Moons Orbiter (JIMO), which will use a nuclear-powered rocket to orbit several of the giant planet's satellites in the course of one mission - impossible using conventional rockets.

All this would be wonderful, but here's where the storm clouds gather. JIMO is likely to far exceed Cassini's \$3.2-billion cost. Its nuclear power plant has yet to be built. It relies on a heavy-lifter launch vehicle that doesn't yet exist. Nuclear power offers shorter trip times to the outer planets and enough onboard electricity to power instruments far more advanced than any previously sent into deep space, but any new technology is likely to suffer cost overruns and delays. The expected launch date has already slipped from 2011 to 2015.

Add to this uncertainty NASA's plan to send astronauts to the Moon and Mars, a shift in direction that - if it survives this year's US presidential election - will affect every other programme at the agency. Nuclear rockets would be useful for human expeditions as well, and optimists say that having two customers makes the programme stronger than if JIMO were its only purpose. Pessimists worry that this expensive planetary mission will be easy to cut if NASA runs into money trouble with its Moon-Mars plans.

International cooperation is another unknown. Cassini is the historic high point of collaboration between European and US planetary scientists. The Huygens probe was built in Europe, which also footed nearly a quarter of the overall project's bill. But such collaboration seems to be on the wane. The Mars programme, once envisaged as truly international, ended up being mostly American. The JIMO mission is, at this point, NASA-only. If European and US space scientists continue to go it alone, exploration of the Solar System will be more expensive for each side.

After the successful Voyager fly-bys of the 1980s, planetary scientists entered the doldrums, with no new science results for nearly a decade. NASA has a plan to avoid such a hiatus in the future. But it depends on following through with key projects such as JIMO, whose political fortunes bear watching even as we sit back and enjoy the spectacular Cassini images of Saturn, its rings and moons.

\section{News cornucopia}

How to access all of Nature publications' journalism in one fell swoop.

$\mathrm{N}$ ow more than ever, people need good news coverage about science. Most media organizations are cutting back on budgets for journalists, including science journalists, who are becoming less and less able to spend time digging into stories, or even searching for them in the first place. Lots of money is being spent on new platforms for disseminating journalism, as the use of news feeds grows and as we head towards electronic replacements for newspapers. But quality, diversity and depth are increasingly in short supply.

This week, Nature and its sister journals strike a blow against this trend. Simultaneous with the publication of this week's issue of Nature, we are pleased to launch our new online news service, news@nature.com. It delivers free daily stories — a revamped version of Nature Science Update, which it replaces. It also provides all the news and other journalistic content provided by Nature, Nature Medicine, Nature Biotechnology, BioEntrepreneur and Nature Reviews Drug Discovery, as well as career news from Naturejobs.
Subscribers to any of the participating journals get all of this online content at no extra charge and before it is printed. Others can subscribe to the news service directly, receiving the free daily service and the rest as premium content. But for an introductory season over the next two months, the entire service is free of charge for all. It can be found at www.nature.com/news.

Quality? We intend it to be of the highest, in keeping with the values of the Nature name. Diversity? Our site will bring you hardhitting daily coverage of all the big developments in research, as well as the science and technology issues that increasingly affect our lives. But there's room too for the scientific fun that lies buried in specialist journals and meetings, and for a more personal view from our team in the form of blogs and columns. And depth? All the journals provide in-depth coverage of their territories issue after issue.

The team that brings you all this is unmatched in its geographical coverage and award-winning talent. We are confident that your supply of the news of science will be greatly enriched by their efforts. 